\section{MOLECULAR SIGNATURE OF NEOANTIGEN-REACTIVE CD4+ AND CD8+ T CELLS FROM METASTATIC HUMAN CANCERS ENABLES PROSPECTIVE ANTITUMOR TCR PREDICTION}

Frank Lowery*, Sri Krishna, Rami Yoseph, Neilesh Parikh, Praveen Chatani, Yong-Chen William Lu, Nikolaos Zacharakis, Paul Robbins, Maria Parkhurst, Steven Rosenberg. NCl, Bethesda, MD, United States

Background Autologous patient $\mathrm{T}$ cells engineered to express antitumor $\mathrm{T}$ cell receptors (TCRs) and chimeric antigen receptors (CARs) have been effective for the treatment of certain cancer types, ${ }^{1-4}$ and tumor neoantigens encoded by cancer-specific mutations have emerged as major targets of CD4+ and $\mathrm{CD} 8+\mathrm{T}$ cells in immune checkpoint blockade (ICB) and in adoptive cell therapy (ACT) ${ }^{5-9}$ However, only a minority of intratumoral $\mathrm{T}$ cells are reactive to cancer antigens while the majority represent bystander cells. ${ }^{10-12}$ Conventional approaches to isolate tumor-reactive $\mathrm{T}$ cells and identify their TCRs from tumors rely on $\mathrm{T}$ cell function and can be impaired due to $\mathrm{T}$ cell exhaustion and dysfunction. ${ }^{13} 14$

Methods We performed single-cell RNA and T cell receptor (TCR) sequencing (scRNA/TCR-seq) on over 46,000 T cells isolated from eleven archival metastatic tumor samples whose primary cancer types included colon, rectal, breast, anal, and melanoma. From these samples, $15 \mathrm{CD} 8+$ and $17 \mathrm{CD} 4+$ neoantigen-reactive TCR clonotypes (NeoTCRs) were known. We then performed transcriptomic clustering of these cells and mapped known NeoTCR clonotypes onto the transcriptomic map. Subsequently we predicted NeoTCRs from prospective metastatic colon cancer samples based on their presence within clusters sharing gene expression with NeoTCR+ clusters in the archival samples.

Results Projecting known NeoTCRs onto the TIL transcriptomic map, we observed 325 total $\mathrm{T}$ cells bearing these NeoTCRs, and the majority $(>80 \%)$ of NeoTCRs were expressed by $\mathrm{T}$ cells within 2 clusters, one CD4+ and one CD8 + , that included by expression of CXCL13, ENTPD1 (CD39), TOX, TIGIT, LAG3, and PDCD1 (PD-1), indicating a dysfunctional state. Reasoning that $\mathrm{T}$ cells sharing phenotypes with those within the NeoTCR clusters could be novel NeoTCRs, we developed gene signatures (NeoTCR4 and NeoTCR8) of CD4 and CD8 NeoTCR+ cells, respectively, and four prospective patients' TIL were analyzed by scRNA/ TCR-seq and scored according to NeoTCR signatures. We expressed predicted NeoTCRs in healthy donor PBL and screened them with antigen presenting cells (APCs) expressing candidate neoantigens. 33/73 predicted NeoTCRs (including both CD4 and CD8) were reactive against patients' tumors or candidate neoantigens.

Conclusions This study enabled successful detection of tumorspecific NeoTCRs in the sequenced TIL of 14/14 patients for whom reactivity was studied. Deconvolution of NeoTCRs from bystander TCRs within the tumor-immune microenvironment represents an important step in the development of personalized immunotherapeutics, and prospective NeoTCR isolation based on TIL transcriptional phenotypes will allow for rapid development of personalized immunotherapy in the form of lymphocytes expressing these tumor-specific TCRs.

Acknowledgements We thank the Surgery Branch TIL Laboratory and clinical team for generating TIL, and patients enrolled in our clinical protocols. Support from CCR Single Cell Analysis Facility was funded by FNLCR Contract HHSN261200800001E. This work utilized the computational resources of the NIH HPC Biowulf cluster (http://hpc.nih. gov). We also thank NIDAP for providing additional computational support and the CCR Genomics Core for next-generation sequencing support

\section{REFERENCES}

1. Robbins PF, Morgan RA, Feldman SA, Yang JC, Sherry RM, Dudley ME, Wunderlich JR, Nahvi AV, Helman LI, Mackall CL, Kammula US, Hughes MS, Restifo NP, Raffeld M, Lee CCR, Levy CL, Li YF, El-Gamil M, Schwarz SL, Laurencot C, Rosenberg SA.Tumor regression in patients with metastatic synovial cell sarcoma and melanoma using genetically engineered lymphocytes reactive with NY-ESO-1. Clin Oncol 2011;29:917-924.

2. Morgan RA, Dudley ME, Wunderlich JR, Hughes MS, Yang JC, Sherry RM, Royal RE, Topalian SL, Kammula US, Restifo NP, Zheng Z, Nahvi A, de Vries CR, Rogers-Freezer LJ, Mavroukakis SA, Rosenberg SA. Cancer regression in patients after transfer of genetically engineered lymphocytes. Science 2006;314:126-129.

3. June $\mathrm{CH}$, Sadelain M. Chimeric Antigen Receptor Therapy. N Engl J Med 2018;379:64-73.

4. Kochenderfer JN, Yu Z, Frasheri D, Restifo NP, Rosenberg SA. Adoptive transfer of syngeneic $T$ cells transduced with a chimeric antigen receptor that recognizes murine CD19 can eradicate lymphoma and normal B cells. Blood 2010;116:3875-3886.

5. Tran E, Robbins PF, Rosenberg SA, "Final common pathway" of human cancer immunotherapy: targeting random somatic mutations. Nat Immunol 2017; 18:255-262

6. Robbins PF, Lu YC, El-Gamil M, Li YF, Gross C, Gartner J, Lin JC, Teer JK, Cliften $P$, Tycksen E, Samuels $Y$, Rosenberg SA, Mining exomic sequencing data to identify mutated antigens recognized by adoptively transferred tumor-reactive $T$ cells. Nat Med 2013:19:747-752.

7. Parkhurst MR, Robbins PF, Tran E, Prickett TD, Gartner JJ, Jia L, Ivey G, Li YF, ElGamil M, Lalani A, Crystal JS, Sachs A, Groh E, Ray S, Ngo LT, Kivitz S, Pasetto A, Yossef R, Lowery FJ, Goff SL, Lo W, Cafri G, Deniger DC, Malekzadeh P, Ahmadzadeh M, Wunderlich JR, Somerville RPT, Rosenberg SA. Unique Neoantigens Arise from Somatic Mutations in Patients with Gastrointestinal Cancers. Cancer Discov 2019;9:1022-1035.

8. Gubin MM, Zhang $X$, Schuster $H$, Caron E, Ward JP, Noguchi T, Ivanova $Y$, Hundal J, Arthur CD, Krebber WJ, Mulder GE, Toebes M, Vesely MD, Lam SSK, Korman AJ, Allison JP, Freeman GJ, Sharpe AH, Pearce EL, Schumacher TN, Aebersold R, Rammensee HG, Melief CJM, Mardis ER, Gillanders WE, Artyomov MN, Schreiber RD. Checkpoint blockade cancer immunotherapy targets tumourspecific mutant antigens. Nature 2014:515:577-581.

9. van Rooij $N$, van Buuren MM, Philips $D$, Velds $A$, Toebes $M$, Heemskerk $B$, van Dijk $\sqcup A$, Behiati S, Hilkmann $H$, el Atmioui D, Nieuwland M, Stratton MR, Kerk hoven RM, Keșmir C, Haanen JB, Kvistborg P, Schumacher TN. Tumor Exome Analysis Reveals Neoantigen-Specific T-Cell Reactivity in an Ipilimumab-Responsive Melanoma. Journal of Clinical Oncology 2013:31:e439-e442.

10. Duhen $T$, Duhen R, Montler R, Moses J, Moudgil T, de Miranda NF, Goodall CP, Blair TC, Fox BA, McDermott JE, Chang SC, Grunkemeier G, Leidner R, Bell RB, Weinberg AD. Co-expression of CD39 and CD103 identifies tumor-reactive CD8 T cells in human solid tumors. Nat Commun 2018;9:2724.

11. Simoni $Y$, Becht $E$, Fehlings $M$, Loh $C Y$, Koo SL, Teng KWW, Yeong JPS, Nahar $R$, Zhang $T$, Kared $H$, Duan $K$, Ang $N$, Poidinger $M$, Lee $Y Y$, Larbi $A$, Khng $A J$ Tan E, Fu C, Mathew R, Teo M, Lim WT, Toh CK, Ong BH, Koh T, Hillmer AM, Takano A, Lim TKH, Tan EH, Zhai W, Tan DSW, Tan IB, Newell EW, Bystander CD8 T cells are abundant and phenotypically distinct in human tumour infiltrates. Nature 2018;557:575-579

12. Scheper W, Kelderman S, Fanchi LF, Linnemann C, Bendle G, de Rooij MAJ, Hirt C, Mezzadra R, Slagter M, Dijkstra K, Kluin RJC, Snaebjornsson P, Milne K, Nelson BH, Zijlmans H, Kenter G, Voest EE, Haanen JBAG, Schumacher TN. Low and variable tumor reactivity of the intratumoral TCR repertoire in human cancers. Nat Med 2019;25:89-94.

13. Blank CU, Haining WN, Held W, Hogan PG, Kallies A, Lugli E, Lynn RC, Philip M, Rao A, Restifo NP, Schietinger A, Schumacher TN, Schwartzberg PL, Sharpe AH, Speiser DE, Wherry EJ, Youngblood BA, Zehn D. Defining "T cell exhaustion." Nat Rev Immunol 2019;19:665-674.

14. van der Leun AM, Thommen DS, Schumacher TN. CD8 T cell states in human cancer: insights from single-cell analysis. Nat Rev Cancer 2020;20:218-232.

http://dx.doi.org/10.1136/jitc-2021-SITC2021.651 